\title{
A case of crusted scabies combined with bullous scabies
}

\author{
WEN-JING SU, SHENG FANG, AI-JUN CHEN and KUI SHAN
}

Department of Dermatology, The First Affiliated Hospital of Chongqing Medical University, Chongqing 400016, P.R. China

Received September 5, 2014; Accepted June 30, 2015

DOI: $10.3892 /$ etm.2015.2668

\begin{abstract}
Scabies is a contagious skin disease that causes extremely itching. It is a parasitic disease caused by the mite Sarcoptes scabiei and characterized by polymorphous lesions. Vesicular and bullous lesions in cases of scabies are rather rare. Bullous scabies has a pemphigoid presentation. Crusted scabies, also known as Norwegian scabies, is a rare and severe form of the disease. The large number of mites present in and on the skin cause scabies to be highly contagious. The present study reports a case of crusted scabies combined with bullous scabies, the clinical and histopathological manifestations of which mimicked those of bullous pemphigoid. Direct and indirect immunofluorescence test results were negative. Bullae recurred and persisted despite systemic corticosteroids and antihistamine medication. The patient was successfully treated with $10 \%$ sulfur cream and remained free of recurrence during the 12 months of follow-up. Differential diagnosis with bullous pemphigoid and the mechanism of formation of bullae are also discussed.
\end{abstract}

\section{Introduction}

Scabies is an itchy and highly contagious skin disease. It is caused by an infestation by the mite Sarcoptes scabiei. The disease is transmitted via direct skin-to-skin contact, and severe and relentless itching is its predominant symptom. The superficial burrows of scabies are most commonly found on the hands, feet, wrists, elbows, back, buttocks and external genitals (1). The majority of dermatologists are familiar with the diagnosis of typical scabies. However, elderly patients occasionally develop crusted scabies, also known as Norwegian scabies (2). A few cases of bullous scabies resembling bullous pemphigoid have also been reported (3-6). The present study reports the case of an elderly patient who presented with crusted scabies combined with bullous scabies.

Correspondence to: Dr Sheng Fang, Department of Dermatology, The First Affiliated Hospital of Chongqing Medical University, 1 Youyi Road, Chongqing 400016, P.R. China

E-mail: fangshengderm@163.com

Key words: Norwegian, bullous, scabies, pemphigoid

\section{Case report}

A 73-year-old woman developed a generalized itchy papular eruption that particularly affected the trunk, arms and thighs and was present for $>3$ months. Initially, the patient was diagnosed with drug-induced eczema, which was treated with antihistamine and topical corticosteroids. However, the therapeutic effects were unsatisfactory and the intense itching remained. Approximately 3 days prior to admission to the First Affiliated Hospital of Chongqing Medical University (Chongqing, China) bullous lesions started to form and spread gradually on the bilateral thighs. There was no history of similar problems in the patient's family.

Clinical examination identified erythematous or brownish erythematous and scabbed papular lesions on the patient's trunk and limbs (Fig. 1). The skin was generally dry and scaly (Fig. 2). Blisters were present on inner side of her bilateral thighs and some of these were blood blisters (Fig. 3). Nikolsky sign testing of the blisters was negative. The general physical examination was normal. Biopsy of a tense bulla excised from the left thigh revealed the formation of an subepidermal blister. A small amount of cellular infiltration consisting mainly of neutrophils and eosinophils within the blister and in the upper dermis was observed. The results of direct and indirect immunofluorescence tests, aiming to make a differential diagnosis of other skin disorders, such as pemphigus and Bullous pemphigoid, were negative. Laboratory tests demonstrated that the patient had a reduced red blood cell count $\left(3.30 \times 10^{12} / 1\right)$ and hematocrystallin level (105.0 g/l). Liver and kidney function, antinuclear antibody spectrum and HIV tests were normal.

A diagnosis of bullous pemphigoid was made, and the patient was prescribed oral prednisolone (40 mg/day) and topical corticosteroids. However, the symptoms persisted. The therapeutic effects of antihistamines and other antipruritic agents were unsatisfactory. The itching worsened at night. No other abnormalities were detected. Subsequently, direct microscopy of scrapings from papules on the fingers revealed mites and eggs of Sarcoptes scabiei (Fig. 4). The clinical and pathological features, therapeutic response and detection of scabies mites enabled the patient to be diagnosed as having Norwegian scabies combined with bullous scabies. Therefore, the patient was treated with $10 \%$ sulfur cream for $\sim 7$ days, which resulted in a mild amelioration of the papules and pruritus. The patient subsequently made a complete recovery and no recurrence was observed during a 12-month follow-up. The present study was approved by the Ethics Committee of the First affiliated hospital of the Chongqing Medical 


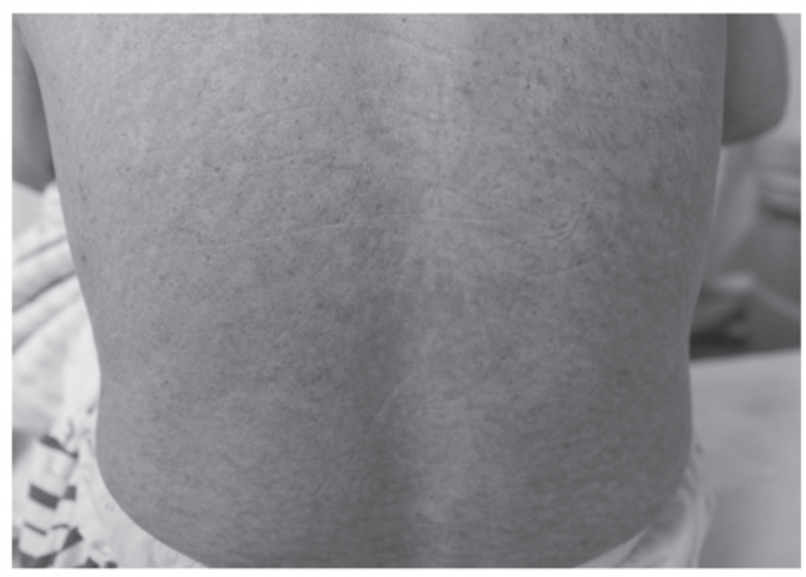

Figure 1. Erythematous and scabby papular lesions on the torso.

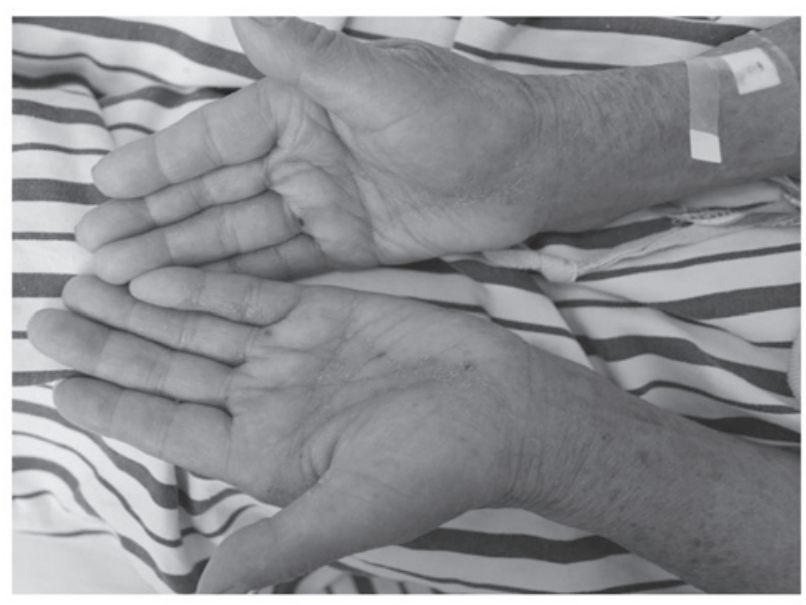

Figure 2. Dry and scaly hyperkeratotic plaques on the palms.

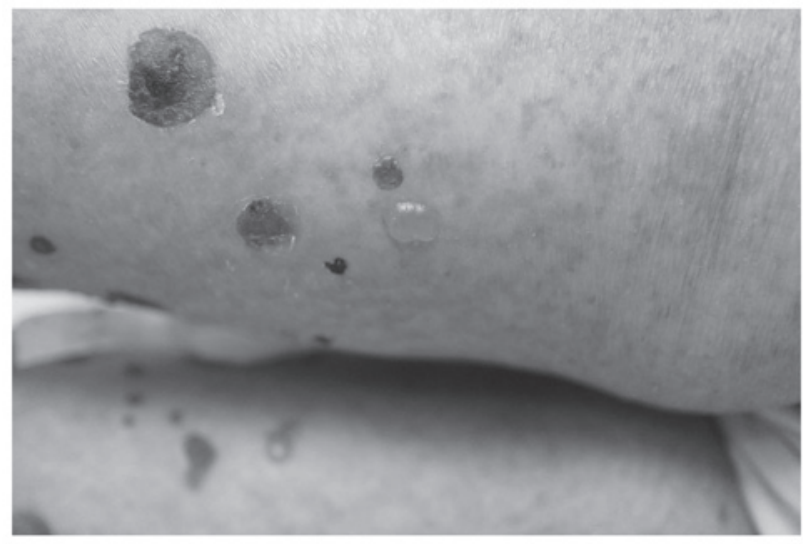

Figure 3. Multiple, discrete, tense bullae on an erythematous base present on the inner bilateral thighs.

University, and written informed consent was obtained from each patient prior to the start of the study.

\section{Discussion}

To the best of our knowledge, there are very few reports concerning Norwegian scabies combined with bullous

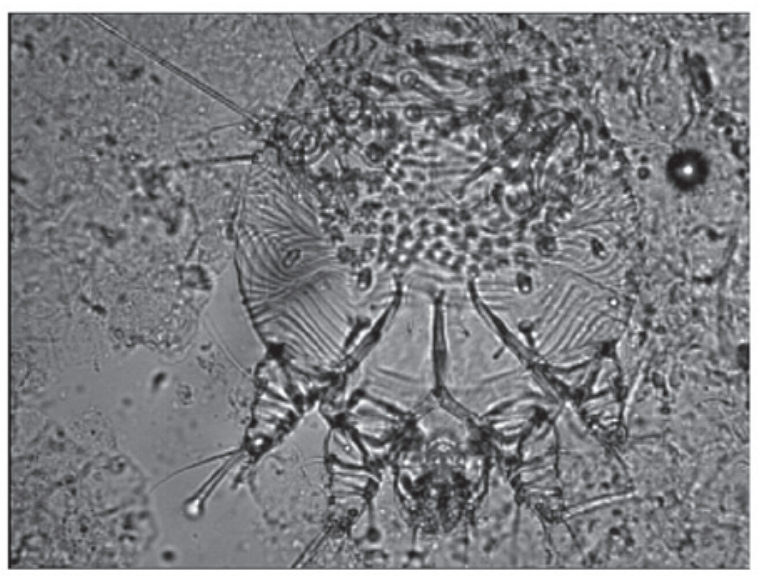

Figure 4. Microscopic examination revealed mites and eggs from the crusted lesions of the fingers. Magnification, x100.

scabies. Scabies is usually clinically divided into common scabies and crusted scabies. Crusted scabies, also known as Norwegian scabies, is a more severe form usually associated with immunosuppression (7). This disorder is very easy to misdiagnose. When it is treated as a drug-induced eruption, eczema or other disease with glucocorticoid, this may exacerbate the scabies and cause blisters to develop from the original skin lesions, as in the present case where the patient was initially treated with prednisolone for eczema and bullous pemphigoid.

Although, scabies may present with a variety of symptoms and signs, patients with scabies rarely present with bullae. Patients with scabies and bullae are often misdiagnosed as having bullous pemphigoid. In fact, clinically, it is extremely challenging to distinguish between bullous pemphigoid and bullous scabies, particularly when skin scrapings do not show any mites or eggs. Light microscopy is also not sufficient to distinguish between these diseases. In the present case, the light microscopy of a blister revealed a subepidermal bulla on the dermoepidermal junction. These results were consistent with bullous pemphigoid. However, there are some differentiating features. Firstly, although bullous scabies can affect individuals of any age, bullous pemphigoid is more common in the elderly. While true bullous pemphigoid always shows linear $\mathrm{C} 3$ or IgG deposition in the basement membrane zone (BMZ), bullous scabies may show linear and granular deposition in the BMZ, particularly when the result of indirect immunofluorescence testing is negative (8). In the present case, the results of direct and indirect immunofluorescence examinations were negative, which is consistent with the diagnosis of bullous scabies. The itchiness and severity of bullous scabies may be worse at night. With bullous scabies, there is sometimes is a family history, since the disease is a contagious infestation. Lastly, bullous pemphigoid usually exhibits a good response to oral prednisolone while bullous scabies can persist and is relieved quickly by antiscabies drugs.

Immunopathogenesis is considered to play an important role in the development of bullous scabies. It has been suggested that the bullous pemphigoid-like eruptions in scabies are caused by the induction of BMZ-reactive 
autoantibodies by scabies mites. Mites may injure the BMZ directly or through their lytic enzymes, resulting in a change in or release of bullous pemphigoid antigen and, subsequently, the initiation of an immunological response with autoantibody production $(9,10)$. Therefore, the deposition of $\mathrm{C} 3$ or IgG in the BMZ can sometimes be observed. Alternatively, a mite component may play an antigenic role that cross-reacts with the bullous pemphigoid antigen resulting in auto-antibody production (11). Some cases test positive for cultures for Staphylococcus aureus. In such cases, bullous lesions might result from superinfection with $S$. aureus, with a mechanism similar to the development of blisters in bullous impetigo $(6,12)$. However, the exact mechanism of bullous lesion development requires further evaluation.

Physicians should be aware of the possibility of bullous scabies in patients who have bullous pemphigoid-like eruptions associated with pruritic papules that show a poor response to steroids. However, scabies infestation-induced bullous pemphigoid should be included in the differential diagnosis. Steroids are ineffective for the treatment of bullous scabies, and classical antiscabieticides are the best treatment options. The present case was successfully treated topically with $10 \%$ sulfur cream. When topical treatment is difficult, ivermectin (200 $\mathrm{mg} / \mathrm{kg}$ orally) is an alternative effective option.

\section{References}

1. Hicks MI and Elston DM: Scabies. Dermatol Ther 22: 279-292, 2009.

2. Kolar KA and Rapini RP: Crusted (Norwegian) scabies. Am Fam Physician 44: 1317-1321, 1991.

3. Nakamura E, Taniguchi $\mathrm{H}$ and Ohtaki N: A case of crusted scabies with a bullous pemphigoid-like eruption and nail involvement. J Dermatol 33: 196-201, 2006.

4. Balighi K, Robati RM and Hejazi N: A dilemma: Bullous-pemphigoid-like eruption in scabies or scabies-induced bullous pemphigoid. Dermatol Online J 12: 13, 2006.

5. Gutte RM: Bullous scabies in an adult: A case report with review of literature. Indian Dermatol Online J 4: 311-313, 2013.

6. Ansarin H, Jalali MH, Mazloomi S, Soltani-Arabshahi R and Setarehshenas R: Scabies presenting with bullous pemphigoid-like lesions. Dermatol Online J 12: 19, 2006.

7. Towersey L, Cunha MX, Feldman CA, Castro CG and Berger TG: Dermoscopy of Norwegian scabies in a patient with acquired immunodeficiency syndrome. An Bras Dermatol 85: 221-223, 2010.

8. Salo OP, Reunala T, Kalimo K and Rantanen T: Immunoglobulin and complement deposits in the skin and circulating immune complexes in scabies. Acta Derm Venereol 62: 73-76, 1982.

9. Veraldi S, Scarabelli G, Zerboni R, Pelosi A and Gianotti R: Bullous scabies. Acta Derm Venereol 76: 167-168, 1996.

10. Kaur S and Thami GP: Bullous scabies in an adult. Clin Exp Dermatol 28: 93-94, 2003.

11. Ostlere LS, Harris D and Rustin MH: Scabies associated with a bullous pemphigoid-like eruption. Br J Dermatol 128: 217-219, 1993.

12. Herman PS: Letter: Scabies and bullae. JAMA 231: 1134, 1975. 